\title{
Design-based strategies for sampling spatial units from regular grids with applications to forest surveys, land use, and land cover estimation
}

\author{
Lorenzo Fattorini $^{\mathbf{a}}$, Piermaria Corona ${ }^{\mathbf{b}}$, Gherardo Chirici ${ }^{\mathrm{c}}$ \\ and Maria Chiara Pagliarella ${ }^{\mathrm{a} *}$
}

\begin{abstract}
The purpose of this paper is to compare some spatial strategies for sampling polygons onto a grid partitioning a study area. Most of the schemes considered in the paper are aimed at avoiding the selection of neighboring polygons. When one or more auxiliary variables are similar or well correlated with the values of the survey variable, the auxiliary information is adopted at estimation level by means of the difference or the regression estimators, or at design level, using the values of auxiliary variables to determine the inclusion probabilities. Applications to large-scale forest inventories, land use estimation, and forest cover estimation are discussed. A simulation study is performed to compare the adopted strategies in terms of bias (if present), accuracy, and accuracy estimation. The simulation is designed to mimic forest inventories and forest cover estimation, starting from some real situations. An application to plan future surveys for land use estimation in Italy is reported. Copyright $@ 2015$ John Wiley \& Sons, Ltd.
\end{abstract}

Keywords: auxiliary information; difference estimator; generalized regression estimator; Horvitz-Thompson estimator; simulation study; spatially balanced sampling

\section{INTRODUCTION}

The wise management of wildlife and agricultural ecosystems strictly depends on the presence of reliable monitoring programs able to provide accurate information on extent, condition, and productivity of natural resources. Estimation of these attributes is a common objective of most programs at a variety of spatial scales. As pointed out by Gregoire and Valentine (2008, p.1), environmental monitoring is mainly based on sample surveys, because "it is impractical, perhaps even impossible, to completely census the entire population of interest without exhausting available resources."

Environmental populations are communities of individuals (e.g., trees) or more complex ecological structures (e.g., forests, streams, woodlots, or tree rows) scattered over a study region. In some cases, the units of an environmental population are sampled by ad hoc schemes such as line intercept sampling, plot sampling, and Bitterlich sampling (e.g., de Vries, 1986; Schreuder et al., 1993; Overton and Stehman, 1995; Thompson, 2002; Gregoire and Valentine, 2008), and the survey variable is recorded for each sampled unit. In other cases, the study area is covered by a grid of regular polygons (e.g., quadrats, rectangles, or hexagons) of adequate size, a sample of polygons is selected from the grid according to a probabilistic sampling scheme, and the amount of the survey variable is recorded within the selected polygons. The main differences between the two approaches are that in the first case sampling is performed on a without-frame population of units, while in the second case, it is performed on a with-frame population of polygons. This paper deals with the second situation.

When sampling polygons from a grid, there is a wide variety of with-frame spatial sampling schemes that are available. The determination of the best strategies to estimate population totals and averages is a challenging issue when the strategies are evaluated from a design-based point of view, that is, when uncertainty only stems from the sampling schemes adopted to perform the surveys. Indeed, in design-based inference the minimal sufficient statistic (i.e., the unordered set of distinct sampled units) is not complete. This lack of completeness leads to a lack of optimal results, in the sense that it is not possible to determine the best estimation strategy, as is customary in model-based approaches (e.g., Thompson, 2002, Chapter 9). Accordingly, no general validity will be claimed about the results achieved in this paper, which should be circumscribed to the situations under which they are obtained or extended, at most, to similar, related cases.

When sampling spatial units, the achievement of a so-called spatially balanced sample (SBS), that is, a sample in which units are well spread throughout the study area, has been the main target for a long time. In most situations, nearby units are more similar than units far

* Correspondence to: M. C. Pagliarella, Department of Economics and Statistics, University of Siena, Siena, Italy. mariachiara.pagliarella@unisi.it

a Department of Economics and Statistics, University of Siena, Siena, Italy

b Consiglio per la Ricerca in Agricoltura e l'Analisi dell'Economia Agraria, Forestry Research Centre, Arezzo, Italy

c Department of Agricultural, Food and Forestry Systems, University of Firenze, Firenze, Italy 